of the laboratories of Sandia and Philips (Amsterdam). These authors have related the thermal, chemical and electrical effects occurring in semiconducting devices to the materials and growth processes used in their fabrication. There are chapters on metal/metal interdiffusion, electromigration in thin films, electrode/ compound substrate reactions, depth analysis and thin film deposition.

I read the chapters on atom transport and chemical reactions with interest because of their topical nature and because I was least well acquainted with their subjects. A new micrometallurgy is introduced in which the conditions for grain boundary diffusion, intermetallic and silicide formation and electromigration in a variety of film materials is treated over depth and surface dimensions of a fraction of a micrometer. As is essential in a book of this kind the text is well illustrated with micrographs.

I did not expect a detailed description of deposition techniques but a balanced and informed account of those in common use. Thus I question the wisdom of referring to ion beam deposition as a promising new technique while omitting mention of plasma chemical procedures which seem to have an important future in silicon technology. Also the reader is not informed that ion beam deposition is only possible when the self-sputtering yield of the incident ions is below one. Further, without experimental evidence it is unsatisfactory to suggest that ion bombardment may aid epitaxy; the case cited of poolycrystalline carbon is for deposition on a non-crystalline substrate. Gencrally, ion impact results in damage.

Sputtering is listed under collected deposition techniques as having the disadvantages of causing substrate damage and heating. Mention could have been made of sputtering apparatus with crossed electric-magnetic fields (magnetron) to deflect electrons from the substrate and reduce heating. It is an over simplification to lump together under "damage" particle and radiation effects which have different sources and intensities in sputtering plant operating from $10^{-3}$ to $10^{-1}$ torr gas pressure. The treatment of mixed component sputtering is not consistent. In the discussion of depth analysis (p149) it is said correctly that after an induction period components are released at rates proportional to their bulk contents, but on p95 it is stated that this only happens if there is no preferential sputtering to change the target surface composition. Few mixtures can have sputtering yields directly proportional to their contents but preferential sputtering raises the surface content of the less favoured component thereby aiding the latter's emission. As the emission of a component is proportional to the product of its surface content and sputtering yield, an equilibrium is reached under which the emission ratio is equal to the component ratio of the bulk material.

It is always dangerous to refer to a reference by way of another (in this case Chopra for Gillam) as on p95 when discussing the enriched Au layer formed on $\mathrm{AuCu}_{3}$ alloy during Ar sputtering. The enriched layer is stable and results in component emission with the bulk contents ratio and not that of the enriched layer as stated on $\mathrm{p} 95$.

The above criticisms are of subsidiary material and they do not detract from

\section{Asiatic ungulates}

Mountain Monarchs: Wild Sheep and Groats of the Himalaya. By George B. Schaller. Pp. 425. (Chicago University Press: Chicago and London, 1978.) $£ 17.50$.

THIS book, the latest in the generally excellent Chicago University Press series on wildlife behaviour and ecology, reports the results of three years of field work with the large mammals of the Himalayas, with emphasis on the wild sheep and goats of the region. The work will undoubtedly stand as the foremost source of information on these Asiatic ungulates for many years. One short chapter describes observations of the large carnivores of the region.

The book is generally organised into chapters dealing with broad biological topics such as distribution, physical attributes, herd dynamics and courtship behaviour. In each chapter, the array of species is treated according to the level of information available. Narrative or tabular summaries of the biological attributes of each species are generally lacking. This fragmented mode of presentation makes the work less readable than other books in the same series. The organisation of the book, together with its enormous geographical scope and the unevenness of information on various species, makes reading frustrating and confusing at times. Nevertheless, the author is to be commended for putting together in one volume the results of years of fieldwork under incredibly difficult conditions and an exhaustive review of the relevant literature, scanty though the latter is. Fortunately, the index is reasonably complete, although it cannot be depended upon to ferret out every piece of information, nor is it very the value of the main text in dealing with the behaviour of metal/semiconductor systems. As a reference work it will be of value to those who want to obtain a comprehensive picture of operational problems in solid-state electronics and also those who are entering the field to produce electronic devices. The authors and the Electrochemical Society (who supported the work) are to be commended for recognising its need and for its useful preparation.

L. Holland

L. Holland was director of $R \& D$ at Edwards High Vacuum, and is now responsible for the Unit for Plasma Materials Processing at the University of Sussex, UK.

helpful in dealing with the vast and confusing array of place-names.

The author concentrated his fieldwork on three species in Pakistan, Nepal and India : urial (Ovis orientalis), wild goat (Capra aegagrus) and bharal or blue sheep (Pseudois nayaur). The information on bharal is probably the most valuable and interesting. This littleknown species possesses a complex mixture of sheep-like and goat-like behavioural and morphological characteristics. Schaller concludes (p44) that, "In general, bharal are considered aberrant goats, with sheep-like affinities". Again, however, the reader must refer to many pages of text, scattered throughout the book, to construct a cohesive picture of the biology of this species.

A concluding chapter on the evolution of the Caprinae provides a new perspective on this topic based on the author's own data on Asiatic caprids and literature review. This discussion of evolution and dispersal presents some counterpoints and alternative hypotheses to those of V. Geist (Mountain Sheep, Chicago University Press, 1971). Taken together, the two books provide a provocative basis for further research. However, as the author makes abundantly clear in his concluding remarks on the conservation of the Himalayan fauna, time is running out. Many species have been fragmented into small isolated populations, occupying habitats drastically altered by man. The reader is left with the clear impression that even the best system of reserves in the Himalayas will be inadequate unless broad changes in land-use practices can be instituted. In this regard, the enormity of the problems, economic, ecological and political, to be faced is evident.

Peter C. Lent

Peter C. Lent is with the US Fish and Wildlife Service, Anchorage, Alaska. 